\title{
Laparoscopic cholecystectomy for acalculous cholecystitis in a neutropenic patient after chemotherapy for acute lymphoblastic leukemia
}

\author{
Konrad Pielaciński ${ }^{1}$, Anna Ejduk², Tadeusz Wróblewski ${ }^{3}$, Andrzej B. Szczepanik ${ }^{1}$ \\ ${ }^{1}$ Department of General and Hematological Surgery, Institute of Hematology and Transfusion Medicine, Warsaw, Poland \\ ${ }^{2}$ Department of Hematology, Institute of Hematology and Transfusion Medicine, Warsaw, Poland \\ ${ }^{3}$ Chair and Department of General, Transplant and Liver Surgery, Medical University of Warsaw, Warsaw, Poland
}

Videosurgery Miniinv 2014; 9 (3): 468-472

DOI: $10.5114 /$ wiitm.2014.45397

\begin{abstract}
Acute acalculous cholecystitis (ACC) is most frequently reported in critically ill patients following sepsis, extensive injury or surgery. It is rather uncommon as a chemotherapy-induced complication, which is usually life-threatening in neutropenic patients subjected to myelosuppressive therapy. A 23-year-old patient with acute lymphoblastic leukemia was subjected to myelosuppressive chemotherapy (cyclophosphamide, cytarabine, pegaspargase). After the first chemotherapy cycle the patient was neutropenic and feverish; she presented with vomiting and pain in the right epigastrium. Ultrasound demonstrated an acalculous gallbladder with wall thickening up to $14 \mathrm{~mm}$. The ACC was diagnosed. Medical therapy included a broad spectrum antibiotic regimen and granulocyte-colony stimulating factors. On the second day after ACC diagnosis the patient's general condition worsened. Laparoscopic cholecystectomy was performed. The resected gallbladder showed no signs of bacterial or leukemic infiltrates. The postoperative course was uneventful. In the management of neutropenic patients with ACC surgical treatment is as important as pharmacological therapy.
\end{abstract}

Key words: laparoscopic cholecystectomy, acute acalculous cholecystitis, acute lymphoblastic leukemia, chemotherapy.

\section{Introduction}

Acute acalculous cholecystitis (ACC) is most frequently diagnosed in critically ill patients after trauma, sepsis, extensive injury or surgery. Also well acknowledged are other disorders and clinical conditions responsible for ACC, which include long-term fasting and/or parenteral feeding, resuscitation, circulatory failure, atherosclerosis, diabetes, solid malignant tumors, proliferative circulatory diseases as well as immunosuppressive disorders (AIDS) and myelosuppressive chemotherapy [1-6].

We present a case of a neutropenic patient with ACC following chemotherapy for acute lymphoblastic leukemia.

\section{Case report}

A 23-year-old patient with normal karyotype pre-B acute lymphoblastic leukemia and no molecular risk factors during the second complete remission following the FLAM protocol chemotherapy (fludarabine phosphate, cytarabine, mitoxantrone hydrochloride) was subjected to the first consolidation treatment according to the PALG ALL6 protocol (cyclophosphamide, cytarabine, pegaspargase). On day 2 of the first cycle she presented with fever $\left(39^{\circ} \mathrm{C}\right)$, abdominal pain and diarrhea on the next day. Piperacillin/tazobactam, fluconazole and nifuroxazide were administered and after $24 \mathrm{~h}$ the temperature returned to normal; after the next 6 days

\section{Address for correspondence}

Konrad Pielaciński MD, Department of General and Hematological Surgery, Institute of Hematology and Transfusion Medicine,

14 Indiry Gandhi St, 02-776 Warsaw, Poland, phone: +48 2234962 68, e-mail: konpiel@tlen.pl 
the symptoms receded and her condition improved. On day 8 the symptoms recurred: fever $\left(38^{\circ} \mathrm{C}\right)$, abdominal pain and diarrhea. Additional tests demonstrated a decrease in blood count parameters with granulocytopenia (WBC 0.1-0.0 G/l). Test results excluded bacteria in peripheral blood, neutropenic enterocolitis (abdominal ultrasound) as well as pseudomembranous enteritis (no Clostridium difficile in feces). Nevertheless, medical therapy was modified to include a broad spectrum antibacterial and antifungal regimen (meropenem, metronidazole, itraconazole) as well as granulocyte-colony stimulating factors (G-CSF) (filgrastim). After 5 days of medical therapy the temperature returned to normal and abdominal pain subsided but nifuroxazide and G-CSF were continued for the next 4 days because of agranulocytosis. Four asymptomatic days followed so the delayed second cycle of chemotherapy was introduced (on day 18 of the first chemotherapy cycle). On the second day (the second chemotherapy cycle) the patient again presented with fever $\left(39^{\circ} \mathrm{C}\right)$ and abdominal pain followed by diarrhea. Candida albicans was discovered in feces culture and the metronidazole medication administered since day 8 was then supplemented with ketoconazole and piperacillin/tazobactam as well as vancomycin and granulocyte-colony stimulating factors when agranulocytosis and Enterobacter cloacae were detected in peripheral blood. Improvement of the general condition was observed as well as a marked reduction of abdominal pain. However, on day 9 the patient again presented with fever accompanied by vomiting and acute pain in the right epigastrium. Abdominal ultrasound demonstrated acalculous gallbladder with overdistention, wall thickening (up to 14 $\mathrm{mm}$ ) and pericholecystic fluid (Photo 1). Acalculous cholecystitis was diagnosed and the medical therapy continued (broad spectrum antibiotic regimen, G-CSF, intravenous fluids, fasting). Nevertheless, the patient's condition deteriorated and on the next day symptoms of renal and liver failure were observed. The patient was qualified for life-saving surgery. Laparoscopic cholecystectomy was performed after two units of platelet concentrate (PC) were transfused and the platelet count increased to $43 \mathrm{G} / \mathrm{l}$. Histopathological evidence demonstrated lymphocyte infiltration of the gallbladder mucous membrane and hyperemia but no presence of neutrophil infiltrations, leukemia cells (TdT-, CD34-), bacteria or fungal cells. Bile culture showed no micro-organisms (bacteria or fungi). The patient was moved to the intensive care ward, where a broad spectrum antibiotic regimen, G-CSF and erythrocyte concentrate (EC) and PC transfusions were continued. On postoperative day 2 the patient presented with fever $\left(38^{\circ} \mathrm{C}\right)$ and lower values of peripheral blood pressure. Chest X-ray demonstrated atelectatic-inflammatory changes (parenchymatous-interstitial) in the lower lung lobes. Intensive respiratory rehabilitation was performed and the imipenem/cilastatin sodium, vancomycin, metronidazole, teicoplanin, ketoconazole medication was supplemented with ciprofloxacin hydrochloride and co-trimoxazole. The therapy was effective; the patient's condition improved. During the next postoperative days the patient manifested no abdominal symptoms related to cholecystectomy, abdominal pain or diarrhea. On postoperative day 4 she was permitted to eat and well tolerated the light diet. On postoperative day 6 a marked improvement was noted in the picture of the peripheral blood. The G-CSF was discontinued as well as antibiotic therapy 2 days later. The patient
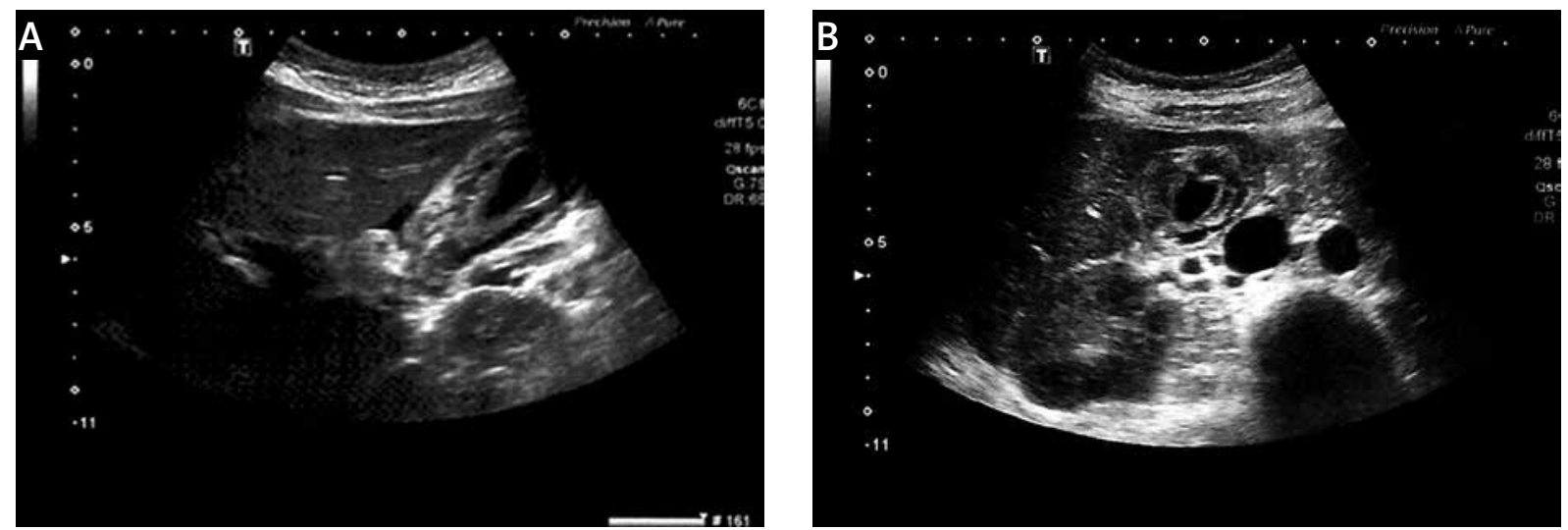

Photo 1. Ultrasound appearance of thickened wall gallbladder 
was transferred to the Department of Hematology and on post-cholecystectomy day 17 she was discharged from hospital in a good general condition.

\section{Discussion}

Chemotherapy-induced complications in patients with myeloproliferative disorders are relatively frequent and directly life-threatening to neutropenic patients. Aggressive chemotherapy is most frequently followed by neutropenic enteritis, while acute acalculous cholecystitis is rare and reported in $0.4 \%$ of patients. In approximately $30 \%$ of them the prognosis is poor due to the severe overall condition induced by the underlying disease $[1-3,7,8]$. The ACC occurs with no deposits and is a local necrotic-inflammatory process mostly ongoing in critically ill patients due to severe systemic disorders related to e.g. trauma, circulatory insufficiency, systemic sepsis or extensive injury [1-4]. Although as yet ACC pathogenesis is not well recognized, gallbladder wall hypoxia is considered as one of its major causative factors. In the course of trauma, systemic sepsis or inflammatory response vasoactive substances (renin-angiotensin) as well as proinflammatory mediators and/or medications (catecholamines) are released into the blood and this induces vasospasms (small arteries in particular) that bring on hypoxia and ischemia reperfusion syndrome. Other etiological factors that are most likely responsible for decreased perfusion of the gallbladder wall and acalculous inflammation include fever-related dehydration and impairment of gallbladder motor functions that induce cholestasis and gallbladder overdistention $[2-4,8]$. Although many studies point to hypoxia/ oxygen deficiency as the major factor in gallbladder wall injury and ACC (regardless of the underlying cause), there are also other acknowledged pathomechanisms of impaired gallbladder integrity. Mucosal epithelium injury may be directly related to bile stasis or cytotoxic medication (chemotherapy) or indirectly to neutropenia or immunosuppression $[1,3,6,7]$. Bacteria in gallbladder are isolated only in about $24 \%$ of neutropenic patients with ACC. In the remaining cases the cultures are usually sterile. This observation is not fully explicable but most authors are of the opinion that pathogenesis of infection in ACC is secondary to hypoxia and even in sepsis induced by various types of pathogens gallbladder necrosis becomes the secondary focus of infection
[1-4]. In the present case we observed several factors that were most likely responsible for ACC. These included chemotherapy, neutropenia, fasting, fever, diarrhea and bacteremia. Neutropenic enterocolitis was suspected when the patient presented chemotherapy-induced neutropenia followed by fever and abdominal pain. The diagnosis was not confirmed but a broad spectrum antibiotic regimen and G-CSF were administered as classic management of feverish neutropenic patients. Complications also occurred after the second cycle of chemotherapy and their course was similar to that of day 9, when the patient reported a different type of abdominal pain and only in the right epigastrium. Medical interview and clinical observations led to the diagnosis of cholecystitis, later confirmed by abdominal ultrasound $[1-7,9,10]$. Early diagnosis of cholecystitis is not easy in critically ill and often unconscious patients undergoing intensive treatment (respiratory therapy, sedation). Such diagnosis may be further prolonged until acute abdominal pain is reported and/or the patient's general condition worsens as a consequence of gallbladder necrosis or perforation [2-5]. The modalities of choice for ACC diagnosis are ultrasound and computed tomography, which reveal the characteristic features of cholecystitis. The most reliable ACC-specific symptom (90-98\% - ultrasound) is gallbladder wall thickened to >3-4 $\mathrm{mm}$ as well as lack of deposits or biliary sludge. The diagnosis is also confirmed by the presence of pericholecystic fluid, gas in the gallbladder wall or intramural edema which presents as a "halo" effect. Despite the high sensitivity and specificity of ultrasound it may provide false positive ACC diagnosis. In cases of "invisible" sludge or shadowless deposits as well as in cases of hypoalbuminemia and ascites there may occur "noninflammatory" thickening and edema of the gall bladder wall [2, 3, 5]. The first-choice surgical procedures in the management of critically ill patients with ACC and considered the least invasive are cholecystostomy (performed under local anesthesia and ultrasound guidance) and laparoscopic cholecystectomy (LCh). The cholecystostomy procedure is effective for about $80 \%$ of patients and the removal of the drainage after 3 weeks usually completes ACC treatment. If no improvement is reported, emergency removal of the gallbladder is indicated. Many authors therefore share the opinion that cholecystectomy and laparoscopic cholecystectomy in particular should be performed immediately after 
ACC diagnosis - even in critically ill patients. Cholecystectomy eliminates the potential infection foci, and reduces the risk of ongoing infection (sepsis) and further complications (multi-organ failure). The LCh is recommended for patients with no absolute contraindications for surgery, especially those with low risk of conversion to open surgery. In cases of severe/acute inflammation such as gall bladder necrosis, the risk of conversion to open surgery is the highest, and therefore open cholecystectomy (OCh) seems to be the best option. In the present case the ultrasound image was suggestive of gall bladder necrosis, and although edema was diagnosed intraoperatively, no technical complications followed and the organ was removed laparoscopically. This confirms the positive opinion about the effectiveness of cholecystectomy in ACC treatment [11-13]. There are authors who do not acknowledge the greater advantage of laparoscopy in this group of patients and report similar incidence rates for complications and mortality for both the cholecystectomy techniques (LCh and OCh). In the case of laparoscopic cholecystectomy, however, not only is trauma reduced but the risk of infection is lower than for the open procedure because the incision wounds and the operation field are smaller. This is especially important in the case of immune-suppressed neutropenic patients, for whom any kind of infection is a threat $[1-3,11-$ 16]. Chemotherapy-induced neutropenia in patients subjected to myelosuppressive therapy is a singularly predisposing factor for life-threatening infections, abdominal infections included. In these cases the primary treatment of choice is a broad spectrum antibiotic regimen, G-CSF, fasting, intravenous hydration and/or total parenteral nutrition as well as close observation of the patient's clinical condition for signs of further complications. Such therapy is often effective and sufficient for neutropenic enterocolitis, while surgery (resection) is reserved for treatment of complications (such as transmural necrosis or intestine perforation). In neutropenic patients with ACC medical therapy should however be quickly supplemented by a surgical procedure adjusted to the patient's general condition. In the case of co-existent choledocholithiasis and cholestasis in particular preoperative endoscopic removal of deposits is first recommended, followed by a selected minimally invasive cholecystectomy technique. Other minimally invasive techniques such as hybrid NOTES and laparoscopy or modifications of the classic 3-4 port ac- cess to the SILS port seem to have less impact on the postoperative course. The choice of surgical technique depends mainly on the patient's clinical condition, while other factors (aesthetic and cosmetic) are secondary. Laparoscopic cholecystectomy is a lesser burden to the patient and ensures the removal of the affected organ. It should therefore be the preferred technique rather than cholecystostomy. The procedure improves the patient's general condition and minimizes the risk of further complications such as multiorgan failure [1-3, 5-7, 10-16].

\section{Conclusions}

In neutropenic patients with ACC intensive medical therapy (a broad spectrum antibiotic regimen) should immediately be supported by a surgical procedure adjusted to the patient's general condition. After removal of the affected gallbladder, which is a potential focus of infection, the ACC symptoms subside and the patient's condition is stabilized. Minimally invasive techniques are preferred in the surgical management of ACC patients as they minimize surgery-related trauma, infection risk and formation of further infection foci.

\section{References}

1. Gorschlüter M, Mey U, Strehl J, et al. Cholecystitis in neutropenic patients: retrospective study and systematic review. Leuk Res 2006; 30: 521-8.

2. Huffman JL, Schenker S. Acute acalculous cholecystitis: a review. Clin Gastroenterol Hepatol 2010; 8: 15-22.

3. Barie PS, Eachempati SR. Acute acalculous cholecystitis. Gastroenterol Clin North Am 2010; 39: 343-57.

4. McChesney JA, Northup PG, Bickston SJ. Acute acalculous cholecystitis associated with systemic sepsis and visceral arterial hypoperfusion: a case series and review of pathophysiology. Dig Dis Sci 2003; 48: 1960-7.

5. Morgan C, Tillett T, Braybrooke J, Ajithkumar T. Management of uncommon chemotherapy-induced emergencies. Lancet Oncol 2011; 12: 806-14.

6. Ullery BW, Pieracci FM, Rodney JR, Barie PS. Neutropenic enterocolitis. Surg Infect 2009; 10: 307-14.

7. Cardona Zorrilla AF, Reveiz Herault L, Casasbuenas A, et al. Systematic review of case reports concerning adults suffering from neutropenic enterocolitis. Clin Transl Oncol 2006; 8: 31-8.

8. Pozo MJ, Camello PJ, Mawe GM. Chemical mediators of gallbladder dysmotility. Curr Med Chem 2004; 11: 1801-12.

9. Aapro MS, Bohlius J, Cameron DA, et al. 2010 update of EORTC guidelines for the use of granulocyte-colony stimulating factor to reduce the incidence of chemotherapy-induced febrile neutropenia in adult patients with lymphoproliferative disorders and solid tumours. Eur J Cancer 2011; 47: 8-32. 
10. Simorov A, Ranade A, Parcells J, et al. Emergent cholecystostomy is superior to open cholecystectomy in extremely ill patients with acalculous cholecystitis: a large multicenter outcome study. Am J Surg 2013; 206: 935-40.

11. Nikfarjam M, Shen L, Fink MA, et al. Percutaneous cholecystostomy for treatment of acute cholecystitis in the era of early laparoscopic cholecystectomy. Surg Laparosc Endosc Percutan Tech 2013; 23: 474-80.

12. Sanjay P, Mittapalli D, Marioud A, et al. Clinical outcomes of a percutaneous cholecystostomy for acute cholecystitis: a multicentre analysis. HPB (Oxford) 2013; 15: 511-6.

13. Bitner M, Jaszewski R, Jander S, Maciejewski M. Laparoscopic cholecystectomy delayed by complicated myocardial infarction with papillary muscle rupture, and performed after unique complex mitral repair. Videosurgery Miniinv 2013; 8: 170-3.

14. Kobiela J, Stefaniak T, Laski D, et al. NOTES. Study on patients' perspective. Videosurgery Miniinv 2013; 8: 232-7.

15. Naumowicz E, Białecki J, Kołomecki K. Results of treatment of patients with gallstone disease and ductal calculi by single-stage laparoscopic cholecystectomy and bile duct exploration. Videosurgery Miniinv 2014; 9: 179-89.

16. Pędziwiatr M, Matłok M, Major P, et al. Laparoscopic surgery of the spleen through single umbilical incision. Videosurgery Miniinv 2013; 8: 8-12.

Received: 4.08.2014, accepted: 20.08.2014. 